\title{
Estado, uso y gestión de la torre defensiva de San Andrés (Santa Cruz de Tenerife)
}

\section{Elena María Pérez González ${ }^{a}$, Juan Diego López Arquillo ${ }^{b}$ y Diria Morales Casañas ${ }^{\mathrm{c}}$}

${ }^{a}$ Universidad Europea de Canarias, c/ Inocencio García, 138300 La Orotava, Tenerife. elenamaria.perez@universidadeuropea.es, ${ }^{\text {b} U n i v e r s i d a d ~ E u r o p e a ~ d e ~ C a n a r i a s, ~ c / ~ I n o c e n c i o ~ G a r c i ́ a, ~} 138300$ La Orotava, Tenerife. juandiego.lopez@universidadeuropea.es y

${ }^{\mathrm{c}}$ Facultad de Derecho ULL, camino de la Hornera, 3738205 San Cristóbal de la Laguna, Tenerife. dmoralec@ull.edu.es.

\begin{abstract}
Resumen
La Torre de San Andrés formó parte de una serie de fortificaciones del siglo XVIII para la defensa de la isla de Tenerife (Islas Canarias). Su ubicación estratégica junto a la rambla de San Andrés ha determinado su conservación, provocada por diversas avenidas tormentosas, desde 1706 que se levantara la primera, hasta 1770 en la que se levantaría la tercera y definitiva, y ha llegado hasta nuestros días semicolapsada por la gran avenida de 1895. Este bien cultural ha sido objeto en los últimos años de un debate público entre los vecinos de Santa Cruz de Tenerife, en general, y en particular entre los vecinos del barrio de San Andrés, sobre su uso, tipo de intervención y gestión de la torre. A pesar de las medidas de protección jurídicas aplicadas, actualmente la torre se encuentra en estado de colapso parcial en su sección norte, inscrita dentro de una rotonda de tráfico rodado y aislada del espacio público del núcleo de San Andrés. El enclave ha perdido su relación original con el mar y es evidente su falta de mantenimiento. En este trabajo se analizará el papel de la percepción y la participación social en la gestión de este inmueble y cómo puede determinar su uso y conservación.
\end{abstract}

Palabras clave: gestión del patrimonio cultural, patrimonio militar, fortificaciones, participación social, Tenerife.

\begin{abstract}
The Tower of San Andrés was part of a series of 18th century fortifications for the defense of the island of Tenerife (Canary Islands). Its strategic location next to the Rambla de San Andrés has determined its conservation, caused by several stormy avenues, from 1706 when the first one was built, to 1770 when the third and final one was built, and it has reached our days semi-collapsed by the great avenue of 1895. In recent years, this cultural asset has been the subject of public debate among the residents of Santa Cruz de Tenerife, in general, and among the residents of the San Andrés district, regarding its use, type of intervention and management of the tower. Despite the legal protection measures applied, the tower is currently in a state of partial collapse in its northern section, inscribed within a traffic circle and isolated from the public space of the San Andrés core. The site has lost its original relationship with the sea and its lack of maintenance is evident. This paper will analyze the role of social perception and participation in the management of this building and how it can determine its use and conservation.
\end{abstract}

Keywords: cultural heritage management, fortifications, military heritage, social involvement, Tenerife. 


\section{Introducción}

La torre defensiva de San Andrés forma parte de un amplio sistema defensivo que protegía, desde el siglo XVII, el noreste de la isla de Tenerife, una zona natural de recalada pues resguardaba de los vientos alisios por el macizo de Anaga. Esta es la última fortificación de un territorio que supera los $15 \mathrm{~km}$ que hay entre esta torre, al noreste, y el cierre de la rambla del barranco hondo al suroeste. A pesar del valor histórico de este inmueble y de las medidas de protección jurídicas aplicadas para su protección, la torre se encuentra en la actualidad en un estado de conservación deplorable. Es precisamente el contraste que existe entre estas medidas legales de protección de este inmueble, y las acciones de la población local que reivindican su conservación y uso, la que nos ha llevado a realizar esta investigación y reflexión en torno a la gestión actual de este patrimonio cultural. Por tanto, es nuestro objetivo principal, valorar y aportar una visión o estrategia para una reintegración de la Torre de San Andrés, que aúne los hitos y las variables que hemos detectado en nuestro análisis, poniendo en valor acciones que integren la mirada experta y la de la población local.

Así, el estudio que hemos desarrollado se ha basado en un estudio descriptivo y una reflexión teórica para analizar cómo los gestores de este bien cultural, directos e indirectos, han tratado este patrimonio, lo que nos permitirá poder definir las mejores prácticas o aportar soluciones a su gestión. Hemos realizado un análisis de las metodologías cualitativas realizadas por los colectivos ciudadanos, las acciones legales y aquellas procedentes de las intervenciones de los profesionales y de las instituciones públicas.

Los procesos de participación no han sido habituales en la gestión del patrimonio cultural. Si bien hay un consenso internacional en definir qué es el patrimonio cultural lo cierto es que no lo hay en cuanto a quién debe decidir sobre los usos y la conservación (Castillo, 2019). Por ello, el valor del patrimonio cultural y su proceso social de construcción y gestión a través de métodos como los procesos participativos, ha sido objeto de estudio cada vez más en los últimos años en nuestro país, aportando interesantes visiones y propuestas para su gestión (Castillo y Querol, 2014; Castillo et al., 2016; Durán y Carrera, 2017; García, 2019; entre otros).

La participación activa de los diferentes agentes sociales en la gestión del patrimonio cultural se contempla en la Convención de Faro del año 2005 (Consejo de Europa, 2005), o en el Convenio Europeo sobre Patrimonio Cultural (1992) -ratificado en el año 2018 por España-. En estos documentos se reconoce la participación como una vía para colaborar y trabajar las administraciones, los expertos y la población local en un mismo nivel. Ello genera nuevos espacios de actuaciones en los que las personas pueden activar los bienes culturales, para su uso y protección.

En nuestro caso, hemos detectado que los procesos de participación están jugando un rol muy destacado en la gestión o no gestión- de la Torre de San Andrés en los últimos años, lo que está generando un interesante debate entre la comunidad local, las administraciones públicas y los académicos. Si bien estas acciones no se han materializado en un resultado positivo, pues aún no se ha conseguido intervenir y mejorar la calidad material de la torre y tampoco la del entorno derivado de su estado actual, si han conseguido aumentar el grado de consciencia de la percepción social sobre la necesidad de implementar un modelo de gestión.

Si bien las acciones cívicas que veremos en este trabajo podrían ser consideradas de escasa envergadura, de nulo impacto en la conservación de la torre, lo cierto es que están teniendo una repercusión social importante y constituyendo la base de un importante movimiento social para la defensa de este inmueble. Aun así, debemos poner de manifiesto, la gran brecha que existe entre las actuaciones más oficialistas y las querencias o denuncias de las personas que viven y conviven con el patrimonio cultural.

Por tanto, y como parte de nuestro objetivo, entendemos que los procesos de participación social podrían contribuir a disminuir la brecha a la que nos referimos y que estos procesos, con la ayuda de una correcta identificación de los agentes clave podrían proponer e implementar acciones para proteger este enclave histórico. 


\section{Historia, caracterización y estado actual de la Torre de San Andrés}

La historia de este enclave defensivo se inicia en 1693 con lo que en ese momento es un reducto artillado que debía defender el pueblo de San Andrés y en el que los barcos se refugiaban en sus aguas de los ataques de los piratas. En 1706 ya hay evidencia de una torre levantada por el ingeniero Miguel Tiburcio Rossel, posiblemente por órdenes del Capitán General D. Agustín de Robles Lorenzana, quien ocupó la Capitanía General de Canarias de 1705 a 1709 y al que se le atribuye la promoción de la defensa de la entonces villa y puerto. En 1740 la torre es reedificada por Antonio Riviere, debido al estado en el que se encuentra por las fuertes avenidas de los barrancos en cuya bifurcación se encuentra. En la primavera de 1769 una nueva avenida hace necesario que vuelva a ser reconstruida, esta vez a cargo del ingeniero Alfonso Sánchez Ochando (Tous, 2012). La torre tuvo un papel destacado en el ataque del vicealmirante Horacio Nelson en Julio de 1797, forzando durante el primer ataque el acceso de las tropas inglesas por el barranco de Valleseco -donde las milicias españolas les impidieron el paso- y posteriormente repeliendo el desembarco de tres lanchas en el tercer intento de Nelson, entre el 23 y el 24 de julio de aquel año (Vila, 2005). En 1851, la torre que aún se encontraba en perfecto estado, fue objeto de un estudio e inventario de sus partes y cuerpo de guardia (Tous, 2012). A partir de esa fecha, la torre comienza a sufrir nuevos desperfectos y derrumbes por causa de su comprometida ubicación en la desembocadura de la rambla de San Andrés, en la confluencia de la rambla de las Huertas, en el año 1878 y en el que se decide su desartillado ${ }^{1}$ - años 1893, 1894 y el último, en 1898 que la deja en su estado actual: semicolapsada y con la declaración de ruina. En 1924 es declarada no apta para uso militar y dos años más tarde es cedida al Ayuntamiento de Santa Cruz que la usó -en su parte no colapsada- como calabozo -ente las décadas de los 50 y 70 del siglo XX- y almacén. La torre está declarada Patrimonio Histórico Español por Declaración Genérica del Decreto de 22 de abril de 1949 y pasó a ser automáticamente Bien de Interés Cultural por la Ley 16/1985 de Patrimonio Histórico Español. En 1999, año de aprobación de la Ley de 15 de marzo de Patrimonio Histórico de Canarias -derogada por la actual Ley 11/2019, de 25 de abril, de Patrimonio Cultural- se llevó a cabo la delimitación del entorno de protección de la torre defensiva de San Andrés como Bien de Interés Cultural.

La torre posee planta circular, con paramentos en exviaje hacia el interior y un diámetro principal en base de 17,50 metros -21 varas castellanas- y una altura en coronación virtual de 6,20 metros; conformada en cantería basáltica semirregular y enripiada. Actualmente la torre se encuentra en el estado de colapso parcial en su sección noreste, que prácticamente secciona la torre por la mitad, restando la mitad no colapsada en evidente riesgo por la falta de contrarresto de los empujes de la sección de cubierta no colapsada, que ha pasado a estar en voladizo.

Las secciones colapsadas se encuentran en el lugar y no han sido reaprovechadas ni desplazadas (Fig. 1).
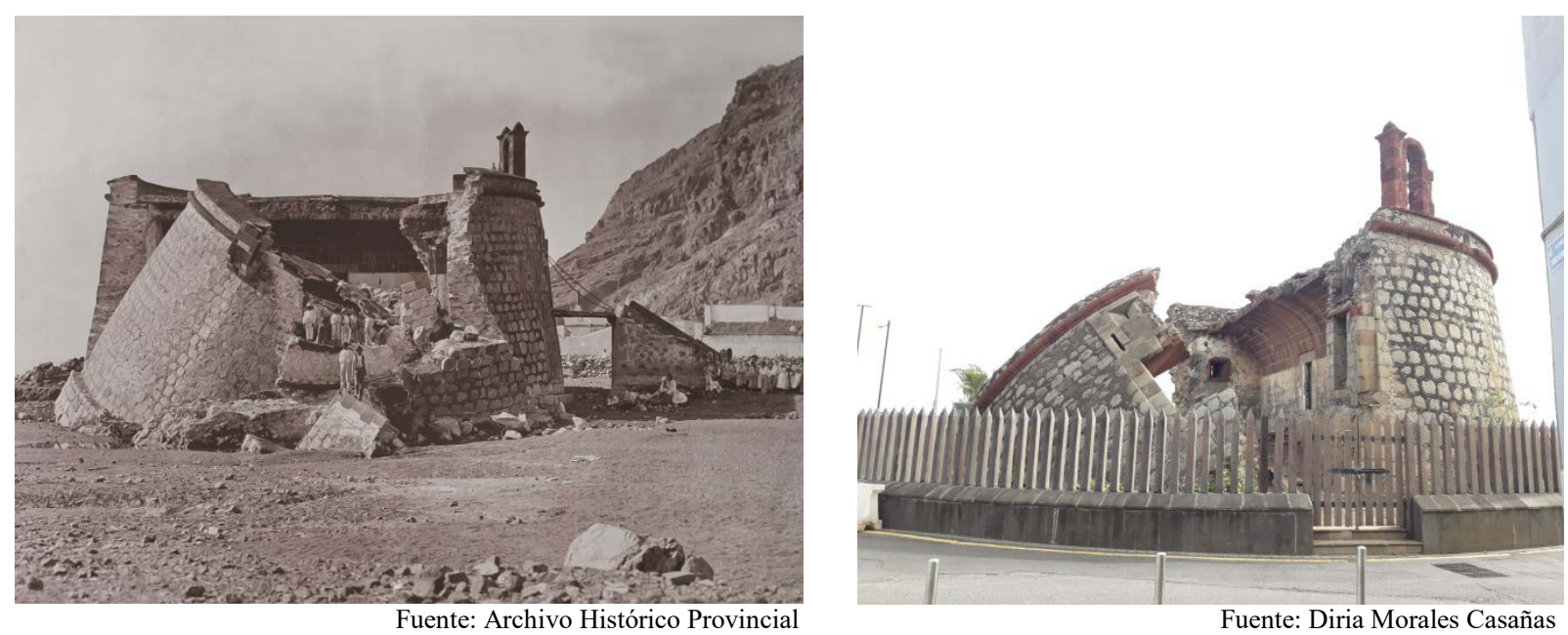

Fig. 1 Fotografía de la Torre tras su colapso (1898) -izquierda- y estado actual -derecha-

\footnotetext{
${ }^{1}$ Con la Real Orden de 25 de julio de 1878 se ordena el desartillado.
} 
A día de hoy, la torre se encuentra inscrita dentro de una rotonda de tráfico rodado y aislada del espacio público del núcleo de San Andrés, por medio de una empalizada permanentemente cerrada ${ }^{2}$; su situación ha hecho desaparecer su relación original con el mar y una evidente falta de mantenimiento caracteriza su estado actual. El cauce del barranco ha desaparecido como amenaza, debido a la reconducción de la rambla en su tramo de salida, fruto del Plan Especial para la playa cercana -Playa de las Teresitas -y que, sin embargo, dejó fuera de su ámbito a la torre y su entorno, perdiendo la oportunidad de contextualizar la torre, si no ya en relación al mar, sí en relación al espacio social del núcleo de San Andrés.

\section{La gestión reciente de la Torre de San Andrés: iniciativas ciudadanas y primeros pasos hacia la participación social}

La Torre de San Andrés es un inmueble que ha estado muy ligado con la historia de su comunidad. El Museo Militar Regional $^{3}$ y la Asociación Tertulia de Amigos del 25 de Julio ${ }^{4}$, han sido dos de los agentes que han motivado y apoyado algunas de las acciones que se han puesto en marcha para reivindicar la protección de la torre. Estas acciones han sido el inicio de una, cada vez más insistente, interacción entre las comunidades y las administraciones para proteger y dar un uso a la Torre de San Andrés.

Tal y como se recoge en el expediente de declaración de Bien de Interés Cultural, en el año $1993^{5}$, la Asociación de Vecinos El Pescador de San Andrés, una de las más representativas y activas de la zona, dirigió un escrito a la Dirección General de Patrimonio Histórico del Gobierno de Canarias, tras haber aparecido en la televisión canaria noticias acerca de la puesta a disposición del Gobierno Central al Gobierno Autonómico de partidas presupuestarias para la restauración de edificios declarados monumentos históricos, como así lo era la torre de San Andrés. Con esta iniciativa esta asociación estuvo intercambiando escritos instando y solicitando la restauración del monumento con todas las administraciones públicas implicadas: Ayuntamiento de Santa Cruz de Tenerife, el Cabildo Insular, el Gobierno de Canarias y la Dirección de Costas del Ministerio de Obras Públicas.

Si bien este proceso duró un año, no consiguió poner en marcha la pretendida restauración.

El 10 de diciembre de 1999, la Tertulia de Amigos del 25 de julio y otras asociaciones cívicas y culturales del pueblo de San Andrés, entre las que estaba la ya mencionada asociación de vecinos organizan una charla coloquio con el título $L a$ reconstrucción del Castillo de San Andrés que según el cartel divulgativo trataría sobre la historia, reconstrucción, financiación y posterior utilización del castillo. En el debate se trataron los argumentos de la Viceconsejería de Cultura del Gobierno de Canarias contrarios a su reconstrucción al afirmar que se trataba de una ruina histórica, ya que durante sus más de doscientos años de existencia sólo cincuenta y siete estuvo en uso ${ }^{6}$. Pero para los participantes y los expertos en la charla, esos datos son erróneos pues diferentes publicaciones posteriores, demuestran que los años de vida útil del inmueble fueron ciento ochenta y uno. Entre las conclusiones extraídas de esta actividad, las opiniones se inclinan hacia al hecho de que la torre debía ser reconstruida tal y como se había hecho con otros inmuebles similares en las Islas Canarias: Torre del Conde en La Gomera, San Cristóbal (San Pedro Mártir en Las Palmas ${ }^{7}$ ) o la torre de Gando en Gran Canaria $^{8}$. También se realizaron propuestas de uso como las de crear un centro de visitantes, de tal manera que ello pudiera dinamizar el barrio.

\footnotetext{
${ }^{2}$ El cerramiento de la torre fue solicitado por la ciudadanía debido a los problemas de inseguridad y drogas, vinculados al uso del recinto.

${ }^{3}$ Hoy es el denominado Centro de Historia y Cultura Militar de Canarias.

${ }^{4} \mathrm{Su}$ constitución es oficial el 9 de noviembre de 1995, dos años antes de que se cumpliera el bicentenario de la victoriosa defensa de Santa Cruz frente al ataque de la flota inglesa, comandada por el almirante Horacio Nelson, y que tiene como objetivo primordial la investigación y la difusión de la Historia y la Cultura de Canarias, por medio del debate y del libre intercambio de ideas. Recuperado de: https://amigos25julio.com/

${ }^{5}$ El expediente se incoa en 1994. En 1999 se declara el entorno de protección.

${ }^{6}$ Informe oficina técnica de la Viceconsejería de Cultura y Deportes del Gobierno de Canarias de 18 de abril de 1990 , en el cual sugiere un estudio en profundidad del bien para realizar una intervención de consolidación de la ruina.

${ }^{7}$ Restaurada en 1999. Todas ellas torres o castillos gemelos, aparte de esos tres, también el del Cotillo y Caleta de Fuste en Fuerteventura y Torre del Águila en Lanzarote.

${ }^{8}$ Restaurada en los años 70 propiciado por la construcción de la segunda pista del aeropuerto e inaugurada en 1982.
} 
En el 2011, la torre vuelve a ser noticia. El periodista Noé Román publica el artículo El alma del castillo es la ruina ${ }^{9}$. En esta publicación, el autor pone de manifiesto el debate existente entre la ciudadanía sobre la reconstrucción o no de la torre. Entre sus argumentos, desgrana la opinión de los expertos arquitectos quienes se inclinan por la recuperación del entorno, pero no en su reconstrucción ${ }^{10}$, lo que desencadena un debate social entre estos y la ciudadanía ${ }^{11}$.

El Plan Especial de Ordenación del frente de Playa de las Teresitas (2016), tenía entre sus acciones una oportunidad para intentar llegar a un consenso social sobre la torre, con la realización de un proceso participativo para orientación en la toma de decisiones técnicas. En este caso, y de forma novedosa, se hizo en coordinación con la Gerencia de Urbanismo y el Servicio de Participación Ciudadana del Ayuntamiento de Santa Cruz de Tenerife y se puso hincapié en la necesidad de contar con diversos espacios participativos ${ }^{12}$.

Uno de los objetivos principales de este proceso participativo era el incorporar la opinión de la ciudadanía a la redacción del Plan Especial para enriquecer así sus contenidos ${ }^{13}$. Durante dicho proceso se prestó interés a las baterías de San Andrés y a los puestos de ametralladoras (alguno de ellos deslizados por la ladera de la montaña), que debían estar integrados en ese espacio de esparcimiento en frente de la playa, pero lamentablemente no pudieron expresar su opinión acerca de la torre, puesto que dentro de esa descontextualización que sufre el inmueble, es separado de los restantes elementos de arquitectura defensiva del Valle de Salazar o del frente de San Andrés, al no incluirse en el Plan Especial.

En el año 2018 el ayuntamiento de Santa Cruz de Tenerife aprueba, publica y pone en marcha una consulta ciudadana sobre la posible actuación en el Castillo de San Andrés, dentro de la Estrategia de Desarrollo Urbano Sostenible e Integrado ${ }^{14}$. En el objeto de la citada consulta, se decía que "la ciudadanía forme parte activa de las decisiones y actuaciones a llevar a cabo en la rehabilitación y posterior uso al que se destine este bien patrimonial'. Así, durante dos semanas (entre el 29 de marzo y el 15 de noviembre de ese año), y sólo por medios telemáticos, las personas podían responder a cinco preguntas sobre el inmueble. En la propia información que aparecía en la página web del ayuntamiento, se afirmaba que las respuestas más votadas de las cuatro primeras preguntas, fijarían la naturaleza y las características principales de la intervención que se realizaría en la torre, quedando la quinta respuesta pendiente de análisis por los técnicos municipales y la celebración de un concurso de ideas en función de su viabilidad técnica o no, en donde se fomentaría la participación de equipos multidisciplinares cuyas actuaciones serían analizadas por un jurado compuesto por técnicos municipales, especialistas en patrimonio histórico y por representantes vecinales del Barrio de San Andrés, y que terminaría con la redacción de un proyecto definitivo y adjudicación de la obra a acometer ${ }^{15}$.

De nuevo, esta consulta ciudadana hizo que se publicasen varios artículos en la prensa local sobre la torre.

\subsection{PC²: Proyecto de Patrimonio Cultural y Participación ciudadana}

A lo largo de todo este recorrido, hay que destacar que un año antes, en el año 2017, se inició un proyecto de patrimonio cultural y participación ciudadana, $\mathrm{PC}^{2}$, el cual tenía como objetivo la implicación de la ciudadanía en la gestión, protección y difusión del patrimonio cultural de Santa Cruz, por medio de un proceso participativo. Obviamente, la Torre de San Andrés, se posicionaría como uno de los temas más tratados en este proyecto.

En efecto, $\mathrm{PC}^{2}$ fue un proyecto activado por el Ayuntamiento de Santa Cruz de Tenerife, impulsado por la Concejalía de Patrimonio Histórico por medio de la herramienta ConRed ${ }^{16}$ (Morales, 2018). Una de las finalidades de este proyecto

\footnotetext{
${ }^{9}$ Periódico La Opinión del 07 de abril de 2011.

${ }^{10}$ Solo el catedrático de Historia del Arte Alberto Darias Príncipe (Universidad de La Laguna), estaba de acuerdo con la reconstrucción.

${ }^{11}$ Se publican diversos artículos en prensa opinando sobre este hecho.

${ }^{12}$ Ese mismo año, 2016, Jose Manuel Ledesma, actual cronista de la ciudad y miembro de la tertulia publica en prensa un artículo sobre la desidia en torno a este monumento.

${ }^{13}$ Plan Especial de Ordenación del Frente de Playa de las Teresitas, Anexo III, Informe del proceso participativo. p. 18 (abril 2016 ).

${ }^{14}$ DUSI. “Anaga en el corazón”, presupuestada con $2.100 .000 €$.

${ }^{15}$ Los resultados de esta encuesta nunca fueron publicados.

${ }^{16}$ Red de Entidades Ciudadanas de Santa Cruz de Tenerife para el fomento de la participación ciudadana, convivencia, igualdad y diversidad.
} 
era el de identificar a las personas que de manera individual o colectiva se dedicasen al estudio y difusión de la historia de sus barrios.

Este proyecto contó con 4 fases: información, debate, aportaciones y devolución. Se implementó en los cinco distritos administrativos y sus barrios que conforman el municipio Santa Cruz. Durante las fases de información y debate, se observó y registró un gran interés por todo el patrimonio cultural en torno a la arquitectura defensiva de la ciudad: castillos, baterías, bunkers, depósitos, murallas, etc., pero también todos aquellos relacionados a esta tipología de patrimonio como, las atalayas de Anaga, los telémetros o el semáforo de Igueste ${ }^{17}$, entre otros.

Destacar que este proyecto no fue considerado en el proceso de participación del año 2018.

En el caso del distrito de Anaga, en las diversas reuniones que se celebraron, siempre se puso a debate la Torre de San Andrés, sobre su uso y gestión. En estas sesiones se aportaron propuestas como las de realizar un centro de interpretación de la zona de Anaga, si la gestión debía ser municipal o vecinal, o si incluso este bien debía ser gestionado bajo el paraguas de la Reserva de la Biosfera del Macizo de Anaga. No obstante, durante la fase de propuestas, no se aportó nada.

Lamentablemente, en la fase de presentación de aportaciones, aunque el tema fue debatido ampliamente no se presentó propuesta alguna, pero quedó constancia que era un tema candente, muy recurrente e importante entre los colectivos y ciudadanía de Anaga y especialmente entre la de San Andrés.

\section{Conclusiones}

La gestión del patrimonio cultural debe ser un proceso estratégico en el que la participación de las comunidades es fundamental. La Torre de San Andrés ha sido testigo de diversas acciones ciudadanas que, a pesar de su esfuerzo, no han conseguido legitimar ciertas actuaciones de las administraciones públicas. Por un lado, las comunidades locales interesadas en la intervención de la torre han tenido como objetivo principal la búsqueda de un beneficio social que mejore la calidad de vida y la conservación del inmueble. Por otro lado, la administración con responsabilidad en su tutela se ha ceñido más a considerar asépticamente la torre como un elemento más dentro de la planificación urbana.

Sin duda se debe alcanzar una situación que consiga balancear los objetivos de uso y gestión de la torre, y también establecer un canal adecuado de comunicación entre las propias asociaciones y también con la administración. En el proceso descrito en este estudio se ha detectado una alta participación social; los efectos, no obstante, podrían repercutir en una falta de convencimiento -como estamos convencidos de que así está sucediendo-de que las propuestas de la población no prosperen y aumenten su desmotivación -como la ausencia de propuestas en el proceso participativo de $\mathrm{PC}^{2}$.

Es evidente que se necesita un orden en las herramientas de participación, pero también una mayor coordinación entre las áreas que implementan estos procesos participativos.

En lo que respecta a la intervención del inmueble, si bien existe una falta de quórum en torno a la actuación en la propia torre -y su uso final- existen diferentes ejemplos de acciones que, alejadas de ser banalizaciones de teorías modernas en la conservación monumental, son hoy ejemplares intervenciones en monumentos con un estado similar al que posee la torre de San Andrés. Así, una intervención arquitectónica ha de pasar por la consolidación de los fragmentos funcionales, la estabilización de los colapsados y la imprescindible rehabilitación funcional del conjunto. Desde estos supuestos de actuación común, diversos proyectos contemporáneos han demostrado eficacia técnica para una conservación preventiva inicial, seguida de una actuación fruto consecuente de un trabajo amplio sobre todos los ámbitos que una intervención en el patrimonio cultural implica (López, 2018). Esto es de especial relevancia en casos de actuaciones sobre arquitecturas en las que el mismo carácter patrimonial queda en entredicho, incluso cuando ellas fueron sensibles a condicionantes previos que integraron en sus proyectos, como señala Martínez (2011) en el caso de intervenciones sobre piezas icónicas del Movimiento Moderno.

\footnotetext{
${ }^{17}$ El semáforo eléctrico de la denominada Atalaya de Anaga, en Igueste, fue uno los veinte primeros que se construyeron en la costa española, por Real Orden el 9 de junio de 1884.
} 
Así, el Museo Kolumba en Colonia (Alemania), de Peter Zumthor, mantiene elementos originales con mantenimiento de los restos consolidados y reconstrucción no idealizada del volumen original con morfología abstracta que reconfigura la combinación de ambos a nuevas funciones. El Museo de San Telmo, en San Sebastián, por Fuensanta Nieto y Enrique Sobejano, han conservado y actualizado el uso mediante la utilización de los fragmentos reconocibles colapsados en su entorno y mediante un completamiento de las fábricas desaparecidas. Otros ejemplos destacables son la muralla nazarí de Granada, realizada por Antonio Jiménez Torrecillas o el Castillo de Matrera en Cádiz, realizada por Carlos Quevedo.

Estas actuaciones solo podrán materializarse con un aumento de la confianza entre las instituciones y las comunidades. Precisamente en este sentido, habla González Sanfiel (2014), sobre la legislación canaria en torno al patrimonio cultural, de la desconfianza al trabajar con las administraciones locales. Es evidente que, hasta que esta situación no se supere, se hagan visibles los agentes y mecanismos para actuar sobre el patrimonio cultural, no se podrá conseguir la corresponsabilidad plena para una buena gestión de las ciudades, de los bienes culturales y de la calidad de vida de las comunidades que los acogen.

\section{Referencias}

Castillo, A., Domínguez, M., y Yáñez, A. (2016). Percepción ciudadana del patrimonio mundial y la arqueología en tres ciudades españolas. Complutum, 27(2), 295-314.

Castillo, A. (2019). Participative processes in cultural heritage management. Methodology and critical results bases on experiences within the Spanish World Heritage context. PCA European Journal of Postclassical archaeologies, 9, 61-76.

Castillo, A., y Querol, M. A. (2014). Archaeological Dimension of World Heritage: From Prevention to Social Implications. En A. Castillo (Ed.), Archaeological Dimension of World Heritage: From Prevention to Social Implications (pp. 1-11). New York: Springer-Verlag.

Consejo de Europa (2005). Convenio de Faro. Recuperado de: https:/www.coe.int/en/web/conventions/full-list//conventions/rms/0900001680083746

Durán Salado, M. I., y Carrera Díaz, G. (2017). La participación social en la documentación del patrimonio cultural. Introducción a la documentación del patrimonio cultural. PH Cuadernos, 30, 298-319.

García Herrera, A., Pérez González, E., Castillo Mena, A., Stendardi, D., García Cruz, J. I., Chávez Álvarez, M. A., Ferrer Román, E., y Cruz González, A. (2019). Percepción social y participación social como estrategias de gestión del Patrimonio Cultural del Caserío de Masca. Cuaderniu, 7, 117-143.

González Sanfiel, A. M. (2014). La legislación canaria sobre Patrimonio Cultural: una visión de conjunto. Revista Patrimonio Cultural y Derecho, 18, 29-82.

Morales, D. (2018). PC $^{2}$ Proyecto de patrimonio cultural y participación ciudadana. PH Boletín del Instituto Andaluz del Patrimonio Histórico, Año 25, 92, 9-10.

López Arquillo, J. D., Sebastián Pardo, E., Arizzi, A., et al. (2018). HMTT: the heritage manager of tourist territories of south Spain as a management tool for the integral cycle of heritage 3.0. En Congreso Rehabend Construction Pathology, Rehabilitation Technology and Heritage Management. Cáceres.

Martínez Medina, A. (2011). Las huellas del tiempo en la arquitectura moderna intervenida. En actas de Conferencia Internacional CAH20thC. Documento de Madrid 2011, Madrid.

Tous, J. (2012). La Torre de San Andrés ¿Merece la pena rescatarla?. San Cristobal de La Laguna: Juan Tous Meliá.

Vila Miranda, C. (2005). El ataque de Nelson a Tenerife: la versión inglesa. Anuario de Estudios Atlánticos, 51, 285-313. 